\title{
Rapidity loss, spin, and angular asymmetries in the scattering of a quark from the color field of a proton or nucleus
}

\author{
Jamal Jalilian-Marian (D) \\ Department of Natural Sciences, Baruch College, CUNY, \\ 17 Lexington Avenue, New York, New York 10010, USA \\ and CUNY Graduate Center, 365 Fifth Avenue, New York, New York 10016, USA
}

(Received 2 January 2020; accepted 15 June 2020; published 7 July 2020)

\begin{abstract}
We calculate the helicity amplitudes for scattering of a quark from both large and small $x$ gluons of a target proton or nucleus using spinor helicity formalism. We show that scattering from large $x$ gluons of the target results in nonzero spin asymmetry at intermediate $p_{t}$ as well as rapidity loss of the projectile quark. We comment on how this can also generate angular asymmetries in particle production in high energy collisions.
\end{abstract}

DOI: 10.1103/PhysRevD.102.014008

\section{INTRODUCTION}

The color glass condensate (CGC) is an effective theory of small $x$ gluons in a proton or nucleus, valid in the limit $x \rightarrow 0$ where QCD cross sections are dominated by small $x$ kinematics and gluon saturation is expected to be the dominant dynamics [1]. Whereas there are strong and tantalizing hints about presence and contribution of gluon saturation dynamics to forward rapidity particle production and dijet angular asymmetry in proton-nucleus collisions at RHIC and the LHC, an unambiguous and definitive interpretation of the data in terms of gluon saturation is still lacking [2]. To help clarify the contribution of gluon saturation to these processes, higher order (in $\alpha_{s}$ ) corrections [3] to various particle production cross sections have been computed which improve the precision of CGC calculations in the small $x$ kinematics, commonly taken to be $x \leq 0.01$.

While higher order corrections are invaluable for precision studies of CGC, they are still limited to the small $x$ kinematics where $x \leq 0.01$. Recalling the kinematic relation between transverse momentum and rapidity of a produced particle in high energy collisions and the Bjorken $x$ of the gluons of the target probed in the scattering given by $x \sim \frac{p_{t}}{\sqrt{s}} e^{-y}$ we see that production of high $p_{t}$ particles is necessarily dominated by large $x$ kinematics. Since the center of mass energy $\sqrt{s}$ of a high energy collision, such as that at RHIC or the LHC, is fixed it is perhaps more useful to think of small vs large $x$ limits

Published by the American Physical Society under the terms of the Creative Commons Attribution 4.0 International license. Further distribution of this work must maintain attribution to the author(s) and the published article's title, journal citation, and DOI. Funded by SCOAP . of the observables rather than the $\sqrt{s} \rightarrow \infty$ limit as is commonly done in CGC formalism. This is also important because the large $x$ kinematics will be a significant part of the phase space of proton/nucleus wave function probed in all proposed electron ion colliders [2]. For the first time it will be possible to experimentally investigate in detail the transition from large to small $x$ dynamics in large nuclei in a transverse momentum region where genuinely nonperturbative QCD effects may not be significant.

Even more significant is perhaps the common estimates of the $x$ kinematics contributing to a production cross section in CGC formalism. Unlike DIS structure functions which can be measured at various $Q^{2}$ at a fixed value of $x$, particle production in proton (nucleus)-proton (nucleus) collisions in the collinear factorization formalism involves a convolution in $x$ and is sensitive to a range of parton $x$ in the target (and projectile). In the small $x$ approximation employed in CGC calculations one assumes the target gluon distribution $x G\left(x, Q^{2}\right)$ is rising so fast with $1 / x$ that one can approximate it as being given by the minimum value of $\times\left(\equiv x_{\min }\right)$ in the convolution, symbolically written as

$$
\int_{x_{\min }}^{1} d x x G\left(x, Q^{2}\right) \cdots \simeq x_{\min } G\left(x_{\min }, Q^{2}\right)
$$

where $\cdots$ stands for the rest of the collinearly factorized cross section. While this kind of an approximation may be fine for making parametric estimates or even for semiquantitative analysis of the data it cannot be expected to be precise as the above approximation disregards contribution of the larger $x(x \geq 0.01)$ kinematics. ${ }^{1}$ Furthermore making

\footnotetext{
${ }^{1}$ For a comparison of the target $x$ kinematics contributing to a given process, compare Fig. 10 in [4] with Fig. 1 in [5].
} 
the small $x$ approximation allows one to use eikonal methods [6] which treat the projectile parton as moving on a straight line and not deflected after multiply scattering from the target. Clearly this cannot be a good approximation when the scattered parton is at high $p_{t}$, i.e., deflected by a large angle.

In [7] we proposed a new approach which aims to include contribution of both small and large $x$ partons of the target to a scattering cross section (see also [8]). The small $x$ gluons of the target are treated via CGC methods while large $x$ gluons of the target are treated as the standard partons of QCD-improved parton model. To do so we calculated the amplitude for multiple scatterings of a quark from a color field $\mathcal{A}^{\mu}=S^{\mu}+A^{\mu}$ where $A^{\mu}=\left(\mathcal{A}^{\mu}-S^{\mu}\right)$ and $S^{\mu}=\delta^{\mu-}$ in light cone gauge. We included multiple scatterings from the soft field $S^{\mu}$, radiated by small $x$ gluons, to all orders but kept only the single scattering contribution from the $A^{\mu}$ field radiated by a large $x$ gluon. This allows exchange of potentially large longitudinal and transverse momentum between the projectile and the target, unlike the CGC formalism where scattering involves exchange of small transverse momenta only and no longitudinal momentum is exchanged.

Here we use spinor helicity formalism [9] to calculate the helicity amplitudes for scattering of a quark on a proton or nucleus target, including both small and large $x$ gluons of the target. We show that the scattering cross section is sensitive to the helicity of the projectile quark and that inclusion of scattering from the large $x$ gluon results in spin asymmetry for quark scattering. Furthermore, there is longitudinal momentum transferred from the projectile to the target which results in projectile rapidity loss, unlike the CGC formalism where the projectile longitudinal momentum remains unchanged. We argue that, coupled to a realistic description of the target geometry, this would also lead to angular asymmetries in particle production in high energy proton-proton (nucleus) collisions.

\section{HELICITY AMPLITUDES}

The amplitude for scattering of a quark projectile on both small and large $x$ gluons of a target proton or nucleus was
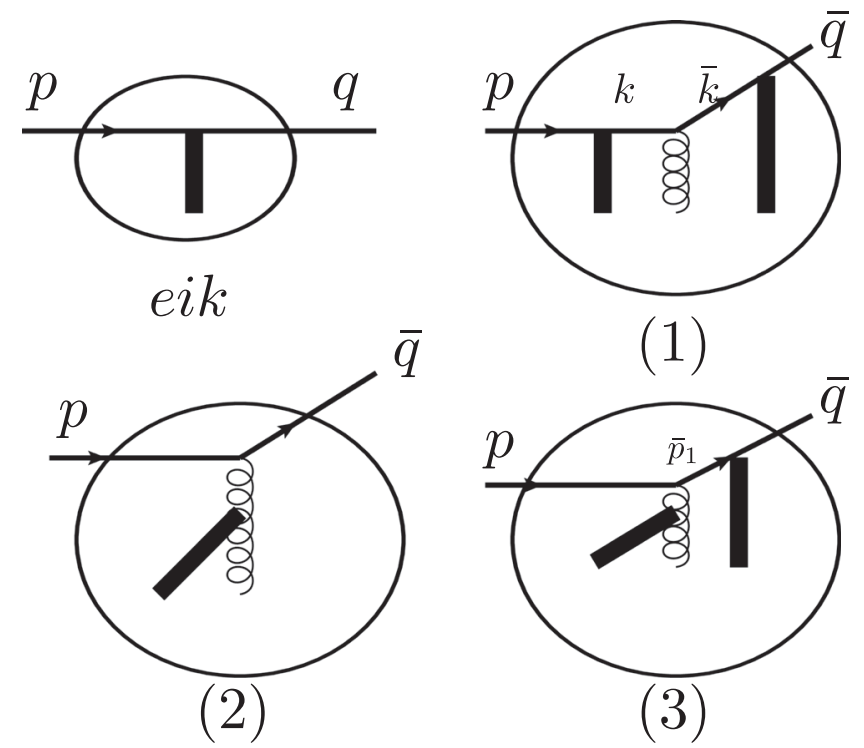

FIG. 1. Scattering of a quark from small and large $x$ gluons of the target.

computed in [7]. Here we use spinor helicity methods to evaluate the amplitude for a given projectile quark helicity. There are in principle four classes of diagrams contributing as shown in Fig. 1. Multiple soft scatterings of the quark (gluon) from the classical color field representing small $x$ gluons of the target is summed into a semi-infinite Wilson line in fundamental (adjoint) representation which is shown as solid black rectangles while the wavy line represents single scattering from a large $x$ gluon of the target proton or nucleus, which itself is shown as a big open ellipse. The eikonal diagram, labeled "eik" above, is the standard diagram used in the hybrid formulation of quark scattering from the small $x$ gluons of a target in the CGC approach [10]. The other three diagrams, labeled 1-3, are nonzero only in the large $x$ kinematics and vanish in the small $x$ limit. The amplitudes are (for details and definitions of Wilson lines see [7], note that anti-path-ordering label is dropped for the sake of compactness)

$$
\begin{aligned}
& i \mathcal{M}_{\mathrm{eik}}(p, q)=2 \pi \delta\left(p^{+}-q^{+}\right) \int d^{2} x_{t} e^{-i\left(q_{t}-p_{t}\right) \cdot x_{t}}\left[V\left(x_{t}\right)-1\right] \mathcal{N}_{\text {eik }} \\
& i \mathcal{M}_{1}(p, q)=\int d^{4} x d^{2} z_{t} d^{2} \bar{z}_{t} \int \frac{d^{2} k_{t}}{(2 \pi)^{2}} \frac{d^{2} \bar{k}_{t}}{(2 \pi)^{2}} e^{i(\bar{k}-k) x} e^{-i\left(\bar{q}_{t}-\bar{k}_{t}\right) \cdot \bar{z}_{t}} e^{-i\left(k_{t}-p_{t}\right) \cdot z_{t}} \bar{V}\left(x^{+}, \bar{z}_{t}\right)\left(i g t^{b}\right) V\left(z_{t}, x^{+}\right) \mathcal{N}_{1}^{b} \\
& i \mathcal{M}_{2}(p, q)=2 i \int d^{4} x e^{i\left(\bar{q}^{+}-p^{+}\right) x^{-}-i\left(\bar{q}_{t}-p_{t}\right) \cdot x_{t}}\left(i g t^{a}\right)\left[\partial_{x^{+}} U^{\dagger}\left(x_{t}, x^{+}\right)\right]^{a b} \mathcal{N}_{2}^{b} \\
& i \mathcal{M}_{3}(p, q)=-2 i \int d^{4} x d^{2} \bar{x}_{t} d \bar{x}^{+} \frac{d^{2} \bar{p}_{1 t}}{(2 \pi)^{2}} e^{i\left(\bar{p}_{1}^{+}-p^{+}\right) x^{-}} e^{-i\left(\bar{p}_{1 t}-p_{t}\right) \cdot x_{t}} e^{-i\left(\bar{q}_{t}-\bar{p}_{1 t}\right) \cdot \bar{x}_{t}}\left[\partial_{\bar{x}^{+}} \bar{V}\left(\bar{x}^{+}, \bar{x}_{t}\right)\right]\left(i g t^{a}\right)\left[\partial_{x^{+}} U^{\dagger}\left(x_{t}, x^{+}\right)\right]^{a b} \mathcal{N}_{3}^{b},
\end{aligned}
$$


where $p, q$ are the momenta of the incoming and outgoing quark. The Dirac structure of the numerators in Eq. (2) is labeled as $\mathcal{N}$, respectively defined as

$$
\begin{aligned}
\mathcal{N}_{\text {eik }} & =\bar{u}(q) \not h u(p) \\
\mathcal{N}_{1}^{b} & =\frac{1}{2 k^{+}} \frac{1}{2 \bar{k}^{+}} \bar{u}(\bar{q})\left[\bar{\not} \overline{\not k} \mathbb{A}^{b}(x) \not k h\right] u(p) \\
\mathcal{N}_{2}^{b} & \left.=\frac{1}{(p-\bar{q})^{2}} \bar{u}(\bar{q})\left[n \cdot(p-\bar{q}) \mathcal{A}^{b}(x)-(p-\bar{q}) \cdot A^{b}(x) \not h\right]\right] u(p) \\
\mathcal{N}_{3}^{b} & =\frac{1}{2 \bar{n} \cdot \bar{p}_{1}\left(p-\bar{p}_{1}\right)^{2}} \bar{u}(\bar{q})\left[\bar{h} \bar{p}_{1}\left(n \cdot\left(p-\bar{p}_{1}\right) \mathbb{A}^{b}(x)-\left(p-\bar{p}_{1}\right) \cdot A^{b}(x) \not h\right)\right] u(p) .
\end{aligned}
$$

Note that we have not written out the color indices in fundamental representation in order to ensure a compact form for the expressions. The contribution of $\mathcal{N}_{\text {eik }}^{ \pm}$in the eikonal diagram is $\mathcal{N}_{\text {eik }}^{ \pm} \sim p^{+} \sim \sqrt{s}$ and is already included in CGC calculations. Furthermore it has zero overlap with the new noneikonal contributions, therefore we will ignore it from now on and focus on the new terms.

To calculate the helicity amplitudes we consider a quark of a given helicity \pm and write the spinor in terns of helicity eigenstates $u_{ \pm}(p)$ defined via $u_{ \pm}(p) \equiv \frac{1 \pm \gamma^{5}}{2} u(p)$. We then use the fact that due to the presence of $\gamma^{5}$ product of states with wrong helicities are zero, for example, $\bar{u}_{+}(p) u_{+}(k)=0$. Furthermore, any on-shell momentum $\not \varnothing$ can be written as a sum over helicity eigenstates $\not p=u_{+}(p) \bar{u}_{+}(p)+u_{-}(p) \bar{u}_{-}(p)$. The specific helicity amplitudes are then ${ }^{2}$

$$
\begin{aligned}
\mathcal{N}_{1}^{+, b} & =\left\langle\bar{k}^{+}\left|A^{b}(x)\right| k^{+}\right\rangle \\
\mathcal{N}_{1}^{-, b} & =\left\langle\bar{k}^{-}\left|\mathcal{A}^{b}(x)\right| k^{-}\right\rangle \\
\mathcal{N}_{2}^{+, b} & =\frac{1}{(p-\bar{q})^{2}}\left[n \cdot(p-\bar{q})\left\langle\bar{q}^{+}\left|\mathcal{A}^{b}(x)\right| p^{+}\right\rangle-\langle n p\rangle[\bar{q} n](p-\bar{q}) \cdot A^{b}(x)\right] \\
\mathcal{N}_{2}^{-, b} & =\frac{1}{(p-\bar{q})^{2}}\left[n \cdot(p-\bar{q})\left\langle\bar{q}^{-}\left|\mathcal{A}^{b}(x)\right| p^{-}\right\rangle-\langle\bar{q} n\rangle[n p](p-\bar{q}) \cdot A^{b}(x)\right] \\
\mathcal{N}_{3}^{+, b} & =\frac{[\bar{q} \bar{n}]\left\langle\bar{n} \bar{p}_{1}\right\rangle\left[n \cdot\left(p-\bar{p}_{1}\right)\left\langle\bar{p}_{1}^{+}\left|\mathcal{A}^{b}(x)\right| p^{+}\right\rangle-\langle n p\rangle\left[\bar{p}_{1} n\right]\left(p-\bar{p}_{1}\right) \cdot A^{b}(x)\right]}{2 \bar{n}^{2} \cdot \bar{p}_{1}\left(p-\bar{p}_{1}\right)^{2}} \\
\mathcal{N}_{3}^{-, b} & =\frac{\langle\bar{q} \bar{n}\rangle\left[\bar{n} \bar{p}_{1}\right]\left[n \cdot\left(p-\bar{p}_{1}\right)\left\langle\bar{p}_{1}^{-}\left|\mathcal{A}^{b}(x)\right| p^{-}\right\rangle-\left\langle\bar{p}_{1} n\right\rangle[n p]\left(p-\bar{p}_{1}\right) \cdot A^{b}(x)\right]}{2 \bar{n} \cdot \bar{p}_{1}\left(p-\bar{p}_{1}\right)^{2}} .
\end{aligned}
$$

We note that

$\mathcal{N}_{3}^{+, b}=\frac{[\bar{q} \bar{n}]\left\langle\bar{n} \bar{p}_{1}\right\rangle}{2 \bar{n} \cdot \bar{p}_{1}} \mathcal{N}_{2}^{+, b}\left(\bar{q} \rightarrow \bar{p}_{1}\right)=\mathcal{N}_{2}^{+, b}\left(\bar{q} \rightarrow \bar{p}_{1}\right)$

and similarly for $\mathcal{N}_{3}^{-, b}$. The helicity amplitudes above (4) are related via identities like

$$
\begin{gathered}
\left\langle\bar{k}^{+}\left|\mathscr{A}_{b}\right| k^{+}\right\rangle=\left\langle k^{-}\left|\mathbb{A}_{b}\right| \bar{k}^{-}\right\rangle \\
\left\langle\bar{k}^{+}\left|\mathscr{A}_{b}\right| k^{+}\right\rangle=\left(\left\langle\bar{k}^{-}\left|\mathscr{A}_{b}\right| k^{-}\right\rangle\right)^{\star} .
\end{gathered}
$$

\footnotetext{
${ }^{2}$ The superscript \pm refers to helicity of the incoming quark. Since helicity is conserved the helicity of the outgoing quark is the same as the helicity of the incoming quark and is not shown explicitly.
}

Using these relations we note that

$$
\mathcal{N}_{1,2,3}^{-, b}=\left[\mathcal{N}_{1,2,3}^{+, b}\right]^{\star}
$$

\section{A. Evaluating the helicity amplitudes}

To evaluate the helicity amplitudes above we will need the following relations:

$$
\left\langle k_{1}^{ \pm}\left|\gamma^{+}\right| k_{2}^{ \pm}\right\rangle=\sqrt{2 k_{1}^{+} 2 k_{2}^{+}}
$$

and 
$\left\langle k_{1}^{ \pm}\left|\gamma_{i}\right| k_{2}^{ \pm}\right\rangle=\sqrt{k_{1}^{+} k_{2}^{+}}\left\{\frac{k_{1 i} \mp i \epsilon_{i j} k_{1}^{j}}{k_{1}^{+}}+\frac{k_{2 i} \pm i \epsilon_{i j} k_{2}^{j}}{k_{2}^{+}}\right\}$.

It should be noted that our final state quark spinors are labeled by their momenta in a bar-ed frame in which their momenta are all longitudinal while momenta of the initial state quark spinors are in the original center of mass frame. Therefore we need to transform all the final state momenta and spinors and write them in the original frame, this can be formally done using the kinematical generators of the Poincare group in the light front form [11] and light front spinors [9] (note that our normalization of the light front spinors differs from [9] and is the same as [12]). Here we denote by $k_{1}\left(p_{1}\right)$ the momentum vector constructed from the components of $\bar{k}\left(\bar{p}_{1}\right)$ in the original frame. We then have

$$
\begin{aligned}
\mathcal{N}_{1, b}^{+} & =p^{+} \sqrt{\frac{q^{+}}{p^{+}}}\left\{2 A_{b}^{-}(x)-A_{b}^{i}(x)\left[\frac{k_{1 i}-i \epsilon_{i j} k_{1}^{j}}{q^{+}}+\frac{k_{i}+i \epsilon_{i j} k^{j}}{p^{+}}\right]\right\} \\
\mathcal{N}_{2, b}^{+} & =\frac{p^{+}}{q_{\perp}^{2}} \sqrt{\frac{q^{+}}{p^{+}}}\left\{\left(1+\frac{q^{+}}{p^{+}}\right) q_{\perp} \cdot A_{\perp}^{b}(x)+i\left(1-\frac{q^{+}}{p^{+}}\right) \epsilon^{i j} q_{i} A_{j}^{b}(x)\right\} \\
\mathcal{N}_{3, b}^{+} & =\mathcal{N}_{2}^{+, b}\left(q_{i} \rightarrow p_{1 i}\right) \\
& =\frac{p^{+}}{p_{1 \perp}^{2}} \sqrt{\frac{q+}{p^{+}}}\left\{\left(1+\frac{q^{+}}{p^{+}}\right) p_{1 \perp} \cdot A_{\perp}^{b}(x)+i\left(1-\frac{q^{+}}{p^{+}}\right) \epsilon^{i j} p_{1 i} A_{j}^{b}(x)\right\} .
\end{aligned}
$$

To facilitate comparison with the contribution of the eikonal term $\sim p^{+} \sim \sqrt{s}$, we have extracted an explicit factor of $p^{+}$ above. We also note the appearance of imaginary term $i \epsilon^{i j}$ in the helicity amplitudes which will lead to spin asymmetry. Next we square the helicity amplitudes which will be needed for calculation of the scattering cross section. This is straightforward and gives

$$
\begin{aligned}
\left|\mathcal{N}_{1}^{+, b c}\right|^{2}= & 4 p^{+} q^{+} A_{b}^{-}(x) A_{c}^{-}(y)-2 A_{b}^{-}(x)\left[q^{+} l_{\perp}+p^{+} l_{1 \perp}\right] \cdot A_{\perp}^{c}(y)-2 A_{c}^{-}(y)\left[q^{+} k_{\perp}+p^{+} k_{1 \perp}\right] \cdot A_{\perp}^{b}(x) \\
& +k_{1 \perp} \cdot A_{\perp}^{b}(x) l_{\perp} \cdot A_{\perp}^{c}(y)+k_{\perp} \cdot A_{\perp}^{b}(x) l_{1 \perp} \cdot A_{\perp}^{c}(y)+l_{\perp} \cdot A_{\perp}^{b}(x) k_{1 \perp} \cdot A_{\perp}^{c}(y)+l_{1 \perp} \cdot A_{\perp}^{b}(x) k_{\perp} \cdot A_{\perp}^{c}(y) \\
& -\left[k_{\perp} \cdot l_{1 \perp}+l_{\perp} \cdot k_{1 \perp}\right] A_{\perp}^{b}(x) \cdot A_{\perp}^{c}(y)+\frac{q^{+}}{p^{+}}\left[k_{\perp} \cdot A_{\perp}^{b}(x) l_{\perp} \cdot A_{\perp}^{c}(y)-l_{\perp} \cdot A_{\perp}^{b}(x) k_{\perp} \cdot A_{\perp}^{c}(y)+k_{\perp} \cdot l_{\perp} A_{\perp}^{b}(x) \cdot A_{\perp}^{c}(y)\right] \\
& +\frac{p^{+}}{q^{+}}\left[k_{1 \perp} \cdot A_{\perp}^{b}(x) l_{1 \perp} \cdot A_{\perp}^{c}(y)-l_{1 \perp} \cdot A_{\perp}^{b}(x) k_{1 \perp} \cdot A_{\perp}^{c}(y)+k_{1 \perp} \cdot l_{1 \perp} A_{\perp}^{b}(x) \cdot A_{\perp}^{c}(y)\right] \\
& +i \epsilon_{i j}\left[2\left[p^{+} l_{1}^{i}-q^{+} l^{i}\right] A_{b}^{-}(x) A_{c}^{j}(y)-2\left[p^{+} k_{1}^{i}-q^{+} k^{i}\right] A_{b}^{j}(x) A_{c}^{-}(y)\right. \\
& \left.+\left[l^{i} k_{1 \perp}-l_{1}^{i} k_{\perp}+\frac{q^{+}}{p^{+}} l^{i} k_{\perp}-\frac{p^{+}}{q^{+}} l_{1}^{i} k_{1 \perp}\right] \cdot A_{\perp}^{b}(x) A_{c}^{j}(y)+\left[k_{1}^{i} l_{\perp}-k^{i} l_{1 \perp}-\frac{q^{+}}{p^{+}} k^{i} l_{\perp}+\frac{p^{+}}{q^{+}} k_{1}^{i} l_{1 \perp}\right] \cdot A_{\perp}^{c}(y) A_{b}^{j}(x)\right]
\end{aligned}
$$

and

$$
\begin{aligned}
\left|\mathcal{N}_{2}^{+, b c}\right|^{2}= & \frac{q^{+}}{p^{+}} \frac{1}{q_{\perp}^{4}}\left\{\left[\left(4 p^{+} q^{+}\right) q_{\perp} \cdot A_{\perp}^{b}(x) q_{\perp} \cdot A_{\perp}^{c}(y)+\left(p^{+}-q^{+}\right)^{2} q_{\perp}^{2} A_{\perp}^{b}(x) \cdot A_{\perp}^{c}(y)\right]\right. \\
& \left.+i \epsilon^{i j}\left[\left(p^{+}\right)^{2}-\left(q^{+}\right)^{2}\right]\left[q_{i} A_{j}^{b}(x) q_{\perp} \cdot A_{\perp}^{c}(y)-q_{i} A_{j}^{c}(y) q_{\perp} \cdot A_{\perp}^{b}(x)\right]\right\}
\end{aligned}
$$

and

$$
\begin{aligned}
\left|\mathcal{N}_{3}^{+, b c}\right|^{2}= & \frac{q^{+}}{p^{+}} \frac{1}{p_{1 \perp}^{2} p_{2 \perp}^{2}}\left\{\left[\left(p^{+}+q^{+}\right)^{2} p_{1 \perp} \cdot A_{\perp}^{b}(x) p_{2 \perp} \cdot A_{\perp}^{c}(y)\right.\right. \\
& \left.-\left(p^{+}-q^{+}\right)^{2}\left[p_{2 \perp} \cdot A_{\perp}^{b}(x) p_{1 \perp} \cdot A_{\perp}^{c}(y)-p_{1 \perp} \cdot p_{2 \perp} A_{\perp}^{b}(x) \cdot A_{\perp}^{c}(y)\right]\right] \\
& \left.+i \epsilon^{i j}\left[\left(p^{+}\right)^{2}-\left(q^{+}\right)^{2}\right]\left[p_{1 i} A_{j}^{b}(x) p_{2 \perp} \cdot A_{\perp}^{c}(y)-p_{2 i} A_{j}^{c}(y) p_{1 \perp} \cdot A_{\perp}^{b}(x)\right]\right\} .
\end{aligned}
$$


There are also the off-diagonal terms in the amplitude squared which are not written out here to save space and especially since they add nothing qualitatively new to the diagonal terms included here. Using relations (7) it should be clear that all the imaginary terms proportional to $i \epsilon^{i j}$ above will cancel between contribution of positive and negative helicity quarks to the unpolarized scattering cross section. On the other hand, if one is interested in spin asymmetry (in this case the so-called longitudinal double asymmetry)

$$
A_{\mathrm{LL}} \equiv \frac{d \sigma^{++}-d \sigma^{-+}}{d \sigma^{++}+d \sigma^{-+}}
$$

the real terms in the helicity amplitude (squared) cancel and the $i \epsilon^{i j}$ terms will survive and give a nonzero spin asymmetry. The appearance of this nonzero spin asymmetry may seem confusing at first since we have said nothing about the helicity of the partons in the target which radiate the gluon field from which the quark is scattering. While the exact form of the asymmetry will depend on the assumptions made about the target partons, it is clear that the color field $A^{\mu}$ radiated by the target partons (large $x$ partons in our case) will "know" about helicity of the target parton which is radiating it, as is illustrated in [13] [see Eqs. (19) and (20)]. Subeikonal corrections at small $x$ are known to contribute to various spin observables [14]. In our case, however, this spin asymmetry is generated at intermediate-large $x$ corresponding to larger transverse momentum of the scattered quark. This can be easily checked by considering the single (large $x$ ) gluon exchange limit of our results, analogous to quark antiquark scattering considered in Appendix A of the last listing of [14].

Furthermore, noneikonal corrections to high energy scattering at small $x$ have been shown to generate angular asymmetries in particle production at small $x$ [15]. Very similarly the large $x$ corrections to eikonal scattering considered here result in angular asymmetries, with the important difference that the asymmetries generated in our formalism will be at higher transverse momenta than the standard small $x$ results. We emphasize that, unlike eikonal scattering, the projectile quark here loses energy (rapidity) which could be used to investigate beam rapidity loss and the limiting fragmentation phenomenon [16] in high energy collisions. Furthermore, our approach allows one to calculate ultrahigh energy neutrino-nucleon cross sections [17] which receive significant contributions from the small $x$ kinematic region but are dominated by a large hard scale $\sim m_{W, Z}$.
There are several issues that need to be investigated further and clarified before one can apply our results to phenomenology. For instance, it is easy to show that all the new noneikonal contributions vanish at small $x$ and one recovers the standard eikonal (tree level CGC) results. Taking the high transverse momentum limit, on the other hand, one is tempted to disregard all the soft multiple scatterings. However this matching with $\mathrm{pQCD}$ must be done carefully [18] in order to avoid difficulties with gauge invariance and needs to be better understood. A study of this limit in DIS structure functions calculated using our results here is in progress.

It will also be interesting to go beyond our tree level process and consider radiation. The simplest case is radiation of a photon from either the initial or final state quark [19]. This would allow one to investigate photon-jet angular correlations from low to high $p_{t}$ (from low to high $x$ ) and study appearance/disappearance of the away side peak and its dependence on the exact kinematics of the process. One can also investigate cold matter energy loss, from incoherent Bethe-Heitler to coherent Landau-PomeranchukMigdal regimes at the same time using our approach. Calculating photon radiation would also serve as a warmup for the calculation of gluon radiation [20] which would then allow one to investigate dijet production and angular correlations in the full transverse momentum range, unlike the CGC calculations where one is limited to the low $p_{t}$ range. Radiation of a real gluon will also be part of the oneloop corrections to our tree level result, which augmented with virtual corrections, would lead to scattering cross sections which generalize and unify the small $x$ expressions of " $k_{t}$-factorized" CGC $[21,22]$ with the collinearly factorized pQCD [23] approach at large $x$.

\section{ACKNOWLEDGMENTS}

We acknowledge support by the DOE Office of Nuclear Physics through Grant No. DE-FG02-09ER41620 and by PSC-CUNY through Grant No. 62185-0050. We would like to thank Centre de Physique Théorique of École Polytechnique for their kind hospitality during the completion of this work and the CNRS for support. We also thank T. Altinoluk, D. Boer, S. Brodsky, M. Hentschinski, W. Horowitz, C. Lorcé, P. Lowdon, C. Marquet, Y. Mehtar-Tani, A. H. Mueller, M. Sievert, W. Vogelsang, and especially L. Dixon and Yu. Kovchegov for very helpful discussions. 
[1] E. Iancu and R. Venugopalan, in Quark Gluon Plasma, edited by R. C. Hwa et al. (World Scientific, Singapore, 2004), pp. 249-3363; F. Gelis, E. Iancu, J. Jalilian-Marian, and R. Venugopalan, Annu. Rev. Nucl. Part. Sci. 60, 463 (2010); J. Jalilian-Marian and Y. V. Kovchegov, Prog. Part. Nucl. Phys. 56, 104 (2006); H. Weigert, Prog. Part. Nucl. Phys. 55, 461 (2005).

[2] E. C. Aschenauer et al., arXiv:1602.03922.

[3] G. A. Chirilli, B. W. Xiao, and F. Yuan, Phys. Rev. D 86, 054005 (2012); Phys. Rev. Lett. 108, 122301 (2012); B. W. Xiao and F. Yuan, arXiv:1407.6314; I. Balitsky and G. A. Chirilli, Phys. Rev. D 77, 014019 (2008); Phys. Rev. D 83, 031502 (2011); Z. B. Kang, I. Vitev, and H. Xing, Phys. Rev. Lett. 113, 062002 (2014); E. Iancu, A. H. Mueller, and D. N. Triantafyllopoulos, J. High Energy Phys. 12 (2016) 041; A. M. Stasto and D. Zaslavsky, Int. J. Mod. Phys. A 31, 1630039 (2016); A. Kovner, M. Lublinsky, and Y. Mulian, J. High Energy Phys. 08 (2014) 114; K. Roy and R. Venugopalan, Phys. Rev. D 101, 034028 (2020).

[4] A. Dumitru, A. Hayashigaki, and J. Jalilian-Marian, Nucl. Phys. A765, 464 (2006).

[5] V. Guzey, M. Strikman, and W. Vogelsang, Phys. Lett. B 603, 173 (2004).

[6] A. Kovner and U.A. Wiedemann, in Quark Gluon Plasma, edited by R. C. Hwa et al. (World Scientific, Singapore, 2004), pp. 192-248; J. Casalderrey-Solana and C. A. Salgado, Acta Phys. Pol. B 38, 3731 (2007).

[7] J. Jalilian-Marian, Phys. Rev. D 96, 074020 (2017); 99, 014043 (2019).

[8] M. Hentschinski, A. Kusina, K. Kutak, and M. Serino, Eur. Phys. J. C 78, 174 (2018); M. Hentschinski, A. Kusina, and K. Kutak, Phys. Rev. D 94, 114013 (2016); O. Gituliar, M. Hentschinski, and K. Kutak, J. High Energy Phys. 01 (2016) 181; I. Balitsky and A. Tarasov, J. High Energy Phys. 06 (2016) 164; 10 (2015) 017.

[9] L. J. Dixon, arXiv:hep-ph/9601359.

[10] A. Dumitru and J. Jalilian-Marian, Phys. Rev. Lett. 89, 022301 (2002); Phys. Lett. B 547, 15 (2002); J. JalilianMarian and Y. V. Kovchegov, Phys. Rev. D 70, 114017 (2004); 71, 079901(E) (2005); C. Marquet, Nucl. Phys. A796, 41 (2007).

[11] K. Y. J. Chiu and S. J. Brodsky, Phys. Rev. D 95, 065035 (2017).

[12] A. Ayala, M. Hentschinski, J. Jalilian-Marian, and M. E. Tejeda-Yeomans, Phys. Lett. B 761, 229 (2016); Nucl. Phys. B920, 232 (2017).
[13] Y. V. Kovchegov, D. Pitonyak, and M. D. Sievert, J. High Energy Phys. 10 (2017) 198.

[14] F. Cougoulic and Y. V. Kovchegov, Phys. Rev. D 100, 114020 (2019); Y. V. Kovchegov and M. D. Sievert, Phys. Rev. D 99, 054032 (2019); Y. V. Kovchegov, D. Pitonyak, and M. D. Sievert, Phys. Lett. B 772, 136 (2017); Phys. Rev. D 95, 014033 (2017); Phys. Rev. Lett. 118, 052001 (2017); J. High Energy Phys. 01 (2016) 072; 10 (2016) 148(E).

[15] P. Agostini, T. Altinoluk, and N. Armesto, Eur. Phys. J. C 79, 790 (2019); Eur. Phys. J. C 79, 600 (2019); T. Altinoluk and A. Dumitru, Phys. Rev. D 94, 074032 (2016); T. Altinoluk, N. Armesto, G. Beuf, and A. Moscoso, J. High Energy Phys. 01 (2016) 114; T. Altinoluk, N. Armesto, G. Beuf, M. Martínez, and C. A. Salgado, J. High Energy Phys. 07 (2014) 068.

[16] J. Jalilian-Marian, Phys. Rev. C 70, 027902 (2004); F. Gelis, A. M. Stasto, and R. Venugopalan, Eur. Phys. J. C 48, 489 (2006).

[17] E. M. Henley and J. Jalilian-Marian, Phys. Rev. D 73, 094004 (2006).

[18] F. Dominguez, C. Marquet, B. W. Xiao, and F. Yuan, Phys. Rev. D 83, 105005 (2011).

[19] F. Gelis and J. Jalilian-Marian, Phys. Rev. D 67, 074019 (2003); 66, 094014 (2002); J. Jalilian-Marian, Nucl. Phys. A753, 307 (2005).

[20] A. Ayala, J. Jalilian-Marian, L. D. McLerran, and R. Venugopalan, Phys. Rev. D 53, 458 (1996).

[21] J. Jalilian-Marian, A. Kovner, L. D. McLerran, and H. Weigert, Phys. Rev. D 55, 5414 (1997); J. Jalilian-Marian, A. Kovner, A. Leonidov, and H. Weigert, Nucl. Phys. B504, 415 (1997); Phys. Rev. D 59, 014014 (1998); Phys. Rev. D 59, 014015 (1998); 59, 034007 (1999); A. Kovner, J. G. Milhano, and H. Weigert, Phys. Rev. D 62, 114005 (2000); A. Kovner and J. G. Milhano, Phys. Rev. D 61, 014012 (1999); E. Iancu, A. Leonidov, and L. D. McLerran, Nucl. Phys. A692, 583 (2001); Phys. Lett. B 510, 133 (2001); E. Ferreiro, E. Iancu, A. Leonidov, and L. McLerran, Nucl. Phys. A703, 489 (2002).

[22] I. Balitsky, Nucl. Phys. B463, 99 (1996); Y. V. Kovchegov, Phys. Rev. D 60, 034008 (1999).

[23] G. Altarelli and G. Parisi, Nucl. Phys. B126, 298 (1977); V. N. Gribov and L. N. Lipatov, Sov. J. Nucl. Phys. 15, 438 (1972); 15, 675 (1972); Yu. Dokshitzer, Sov. Phys. JETP 46, 641 (1977). 